\title{
HST OBSERVATIONS OF THE L1551 IRS5 JET
}

\author{
MALCOLM FRIDLUND \\ Astrophysics Division, Space Science Department \\ European Space Agency \\ ESTEC, P.O. Box 299, NL 2200AG, Noordwijk \\ The Netherlands \\ MONICA HULDTGREN \\ Stockholm Observatory \\ S-133 36, Saltsjöbaden, Sweden \\ AND \\ RENE LISEAU \\ Stockholm Observatory \\ S-133 36, Saltsjöbaden, Sweden
}

\begin{abstract}
The jet emanating from the young stellar object, known as IRS5, located in the molecular cloud L1551 in Taurus has been imaged with the Hubble Space Telescope. The observations and a preliminary interpretation of them is presented. The relevant background history for this important object is briefly reviewed.
\end{abstract}

\section{Introduction \& background}

The L1551-IRS5 Young Stellar Object (YSO) has been in the focus of front line research for the last 20 years or more, at least as what concerns low mass star formation. It is often the first object where a new phenomenon is observed, or where a new technique has been tried out. When we now report the first Hubble Space Telescope (HST) observations, it is also appropriate to briefly review this source. A more general review also exists in the form of the paper of Staude \& Elsässer (1993).

The compact molecular cloud L1551 is located in the southern part of the Taurus molecular cloud region. It shows abundant signs of ongoing star formation, and it has received great attention by those who study this pro- 
cess. It is roughly spherical with a diameter of $1^{\circ}$, located at a distance of $140 \mathrm{pc}$ (Elias 1978, Kenyon et al. 1994) and has an estimated mass of $100 \mathrm{M}_{\odot}$ (Sandqvist \& Bernes, 1980). Optical nebulosity, superposed on the surface of the cloud, is visible on photographic plates of the region. This was classified as a HII region (S239) by Sharpless (1959). The number 1551 is in the listing of Lynds (1962). Felli \& Perinotti (1974) did, however, not detect any radio continuum emanating from this region and suggested that the visible emission emanated from a planetary nebula. Two detached small nebulous knots appear in the compilation by Herbig (1974) as Herbig-Haro objects number 28 and 29. The same objects were noted by Luyten (1963, 1971) to have very high proper motions. In studies of regions containing Herbig Haro objects (Strom et al. 1974, Strom et al. 1976) it was shown, that the S239 nebula possesses the spectroscopic characteristics of $\mathrm{HH}$ objects, and as a consequence this object was 're-designated' HH102. In their $2 \mu \mathrm{m}$ data they found one embedded object associated with the nebulosity - number 5 in their listing (IRS5). A first study of the molecular gas in the region, utilising the $\mathrm{mm}$ radio lines of $\mathrm{CO}$ and ${ }^{13} \mathrm{CO}$ was carried out by Knapp et al. (1976). They found large mass motions as evidenced by very broad line wings, but their spatial resolution was too poor to discern the structure of the flow. They suggested that ordered free-fall collapse of gas onto a newborn stellar cluster was responsible for the broad wings detected in their spectra. Cudworth \& Herbig (1979) did a proper motion study of objects in the region confirming the high velocities of HH28 \& HH29 detected by Luyten $(1963,1971)$. They found that transversal velocities of the order of $150 \mathrm{~km} \mathrm{~s}^{-1}$ were indicated, and even more importantly, that when extending the proper motion vectors backwards in time, they intersected near IRS5 - but at different epochs (timescales of 600 and 2000 years for HH29 and HH28, respectively). Sandqvist \& Bernes (1980) mapped the cloud in the $6 \mathrm{~cm}, 2 \mathrm{~cm}$ and $2 \mathrm{~mm}$ transitions of formaldehyde $\left(\mathrm{H}_{2} \mathrm{CO}\right)$. They estimated the total cloud mass to be $\approx 100 \mathrm{M}_{\odot}$, while they found a very dense core, centered also on IRS5, with a calculated mass of $\geq 0.7 \mathrm{M}_{\odot}$. The same year IRS5 was detected at the far infrared wavelengths of $83 \mu \mathrm{m}$ and $155 \mu \mathrm{m}$ (Fridlund et al., 1980). The total luminosity inferred from these observations was surprisingly low - only $25 \mathrm{~L}_{\odot}$, which suggested that the object was a low mass stellar object in an early stage of evolution. This conclusion - which was confirmed by the near and mid infrared measurements by Beichman \& Harris (1981) - was even more surprising when Snell, Loren and Plambeck (1980) at about the same time detected the first bipolar molecular outflow. This was found centered on IRS5, and these authors found that the outflow was ordered into spatially well separated diametrically opposite lobes - one receeding and one approaching. A lower limit to the mass involved in these motions could also be derived, and the low lu- 
minosity found in the IR observations then immediately indicated that the outflow could not be radiatively driven. Later, IRAS and KAO results increased the bolometric luminosity somewhat to $30 \mathrm{~L}_{\odot}$ to $40 \mathrm{~L}_{\odot}$, but changed neither this conclusion, nor the one that IRS5 is a low mass star (Fridlund et al., 1980).

In a series of studies (Cohen et al. 1982, Bieging et al. 1984, Bieging $\&$ Cohen 1985), radio emission was detected from the immediate vicinity of IRS5 and aligned along the major axis of the CO outflow in a 'jet-like' morphology. It was quite clear given the low luminosity of IRS5 that it could not provide the UV flux necessary to photoionize the gas to a degree sufficient to provide the observed radio continuum. Taken together with the morphology, this indicated instead an origin in shock heated gas.

Cohen et al. (1982) noted the coincidence between the 'radio jet' in the VLA data, and a jet-like object visible on a plate taken by Strom et al. (1974). This object was imaged in detail by Mundt \& Fried (1983), who also detected a number of other optically visible jets, associated with YSO's and with other outflow phenomena. A number of studies now concentrated on detecting IRS5 itself. At one point it was actually believed that the innermost knot visible in the jet was IRS5 itself. It was eventually realised that IRS5 was in fact well hidden behind a very large amount $(\geq 100$ magnitudes) of visual extinction, albeit relatively near the optically visible inner edge of the jet. VLA \& MERLIN observations (Curiel, 1995) show how the knotty structure continues inwards, closer to the source where no optical emission is visible, and the appearence of new radio knots are now being traced into the optical (Curiel, 1995, 1997 private communication; Fridlund $\&$ Liseau 1994). At this time, it was realised, that the reflection nebulosity character of S239 (alias HH102) allowed a possibility of determining the spectroscopic type of IRS5. Mundt et al. (1985) detected P Cygni-profiles of a type observed in FU Orionis type stars, and assigned a spectral type of $\mathrm{G}$ to $\mathrm{K}$, also consistent with IRS5 belonging to this class of objects. Direct $2 \mu \mathrm{m}$ spectroscopy (Carr et al., 1987) provided further evidence of this classification, as well as suggesting the presence of shocked molecular gas very close to IRS5.

Imaging (in the Gunn $\mathrm{r}$ band) of the jet was carried out at three epochs between 1983 and 1987 (Neckel \& Staude, 1987). Their results showed the lifetime of the knots along the jet to be in excess of 4 years, the $V_{t a n}$ for the knot system to be $190 \mathrm{~km} \mathrm{~s}^{-1}$, and a new knot that had appeared at the inner end of the jet during the epoch of the observations. This study was continued by Fridlund \& Liseau (1994), who performed imaging at 3 intervals between 1989 and 1993, thus expanding the total time base to 10 years. These data were collected through $\mathrm{R}, \mathrm{I}, \mathrm{H} \alpha$ and [SII] filters. The images were acquired during seeing conditions between 0.6 arcsec and 


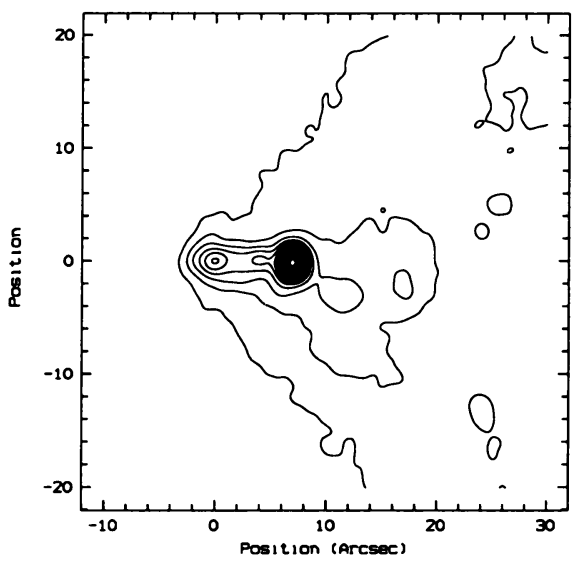

Figure 1. Knots B - F shown on a $\mathrm{R}$ band image. The data was obtained with the Nordic Optical Telescope. This Figure is from Fridlund \& Liseau (1994). Note that the orientation in this Figure is with the jet horizontally and not like it really is - with $17^{\circ}$ inclination to the $\mathrm{E}-\mathrm{W}$ direction.

0.8 arcsec. Although the knots of Neckel \& Staude (1987) were clearly identifiable, their positions had changed significantly (see Figure 1). One knot appeared to be merging with the end point ('working surface') of the jet, and a new knot had appeared, which was also identified in radio data obtained during the same epoch (Curiel, 1995). No less than three transversal velocity systems were detected, and an estimate of the force required to accelerate the individual knots were found to be too small - by two orders of magnitude - than that required to accelerate the bulk of the molecular gas near IRS5 (see Fridlund \& Knee, 1993). This is of course for an ionisation fraction of one.

The present ground based capabilities had thus been brought to its limits, and in order to further study the kinematics - particularly the acceleration/deceleration of individual knots - a multi cycle series of observations with HST appeared well motivated. This is the astrophysical jet closest to the Earth, and thus the shock structure along the jet and at the 'working surface' can be studied in unprecedented detail.

\section{HST Observations}

The area surrounding the L1551 IRS5 jet was imaged using the Wide Field and Planetary Camera 2 (WFPC2) aboard the Hubble Space Telescope (HST) (Trauger et al., 1994). This camera consists of 4 adjacent $800 \times 800$ Loral CCD's. Three of these arrays have an image scale of $0.1^{\prime \prime}$ per pixel, 
while the fourth - the planetary camera (PC) - has an image scale of $0.046^{\prime \prime}$ per pixel. The FOV in this last camera is $25 \times 25 \operatorname{arcsec}^{2}$, and since the jet visible in the ground imagery is less than 15 arcsec long, we centered the PC on the position of IRS5. This will allow us to resolve the smallest elements in any known astrophysical jet. Then, however, we unfortunately loose the multiplexing advantage since with this orientation we can not simultaneously image HH29 or any of the other HH objects within the SW outflow lobe (there are none known within the $\mathrm{NE}$ lobe). We obtained two exposures each through the F656N $(\mathrm{H} \alpha)$ and F673N ([SII] $\lambda 6717,6731 \AA$ ) filters on 1996.093. Total exposure time for each filter was 2500s. On 1996.210 we obtained 3 exposures each through the F675W (R) and F814W (I) filters with total observing times of 1800 s and 2400 s respectively. The data were reduced using methods described e.g. in Holtzman et al., (1995). The calibration of the data into units of $\mathrm{erg} \mathrm{cm}^{-2} \mathrm{~s}^{-1}$ were done according to Holtzman et al., (1995), Heathcote et al., (1996) and the WFPC2 handbook. We compare the results with those of Fridlund \& Liseau (1994) in Table 1.

TABLE 1. Absolute fluxes for knot D and the total jet in this work and in Fridlund \& Liseau (1994 - FL94)

\begin{tabular}{llll}
\hline Feature & $\mathrm{H} \alpha$ & {$[\mathrm{SII}]$} & Source \\
\hline $\mathrm{D}$ & $2.29 \mathrm{E}-14$ & $2.33 \mathrm{E}-14$ & HST \\
$\Sigma_{\text {Alljet }}$ & $5.17 \mathrm{E}-14$ & $4.93 \mathrm{E}-14$ & HST \\
$\mathrm{D}_{\text {Alljet }}$ & $1.8 \mathrm{E}-14$ & $1.9 \mathrm{E}-14$ & FL94 \\
$\Sigma_{\text {A }}$ & 3.34 & $2.9 \mathrm{E}-14$ & FL94 \\
\hline
\end{tabular}

\section{Results}

Figure 1 is a contour plot of part of a R-band image obtained from the ground (Nordic Optical Telescope) in 1989 under 0.6 arcsec seeing conditions (Fridlund \& Liseau, 1994). In this Figure we have identified the features $\mathrm{F}$ to $\mathrm{B}$ from that paper. One can interpret feature $\mathrm{D}$ as constituting a 'working surface' shock at the end of the visible jet. This notion is strengthened by the apparent ellipticity of this feature. At low intensity levels a 'bow-shock' appearance is also clearly visible in such images. In Figure 2 we show greyscale images of the I-band and R-band HST images. The resolution of these images are then 12 times higher than in the best ground based data available. This is also immediately apparent. The 


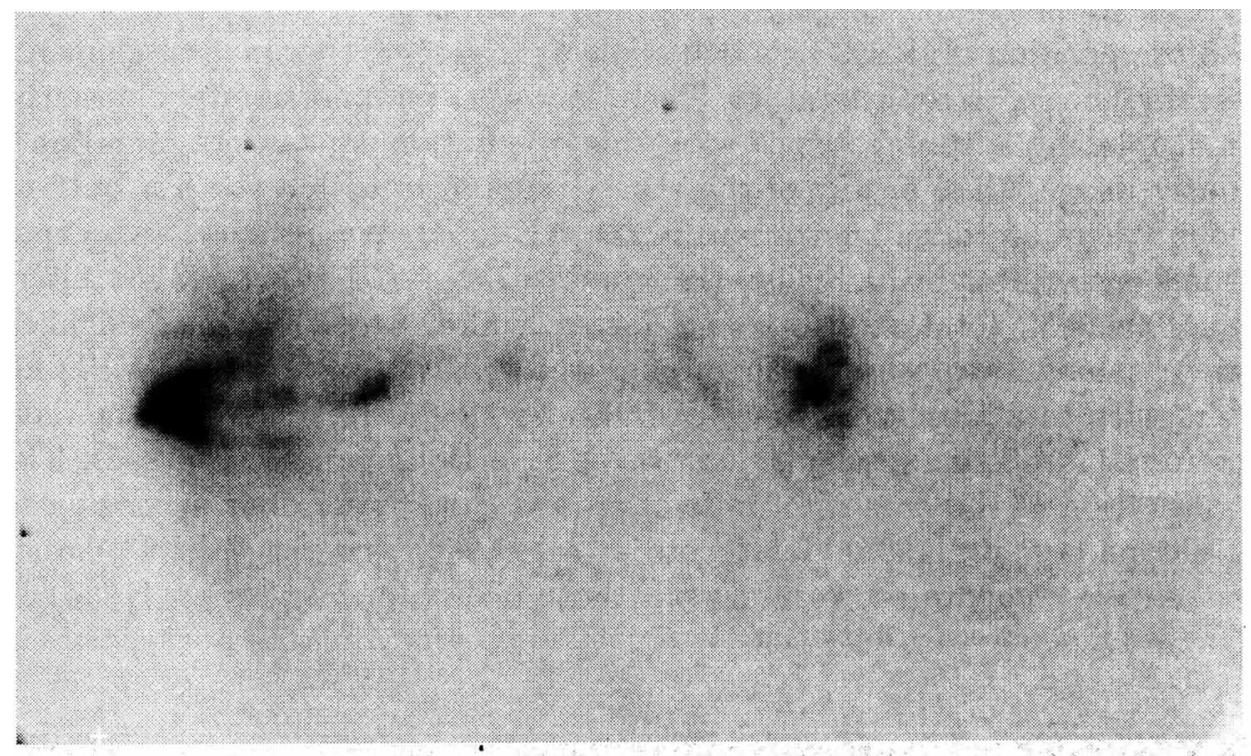

Figure 2. The HST/PC I-band (top) and R-band images. The images have been rotated to match the orientation in Figure 1.

body of the jet itself is much narrower and shows a clear 'wiggling' as it progresses outwards. Features F \& D can be readily identified in the HST data - but with much more substructure. Knot D is very different from 


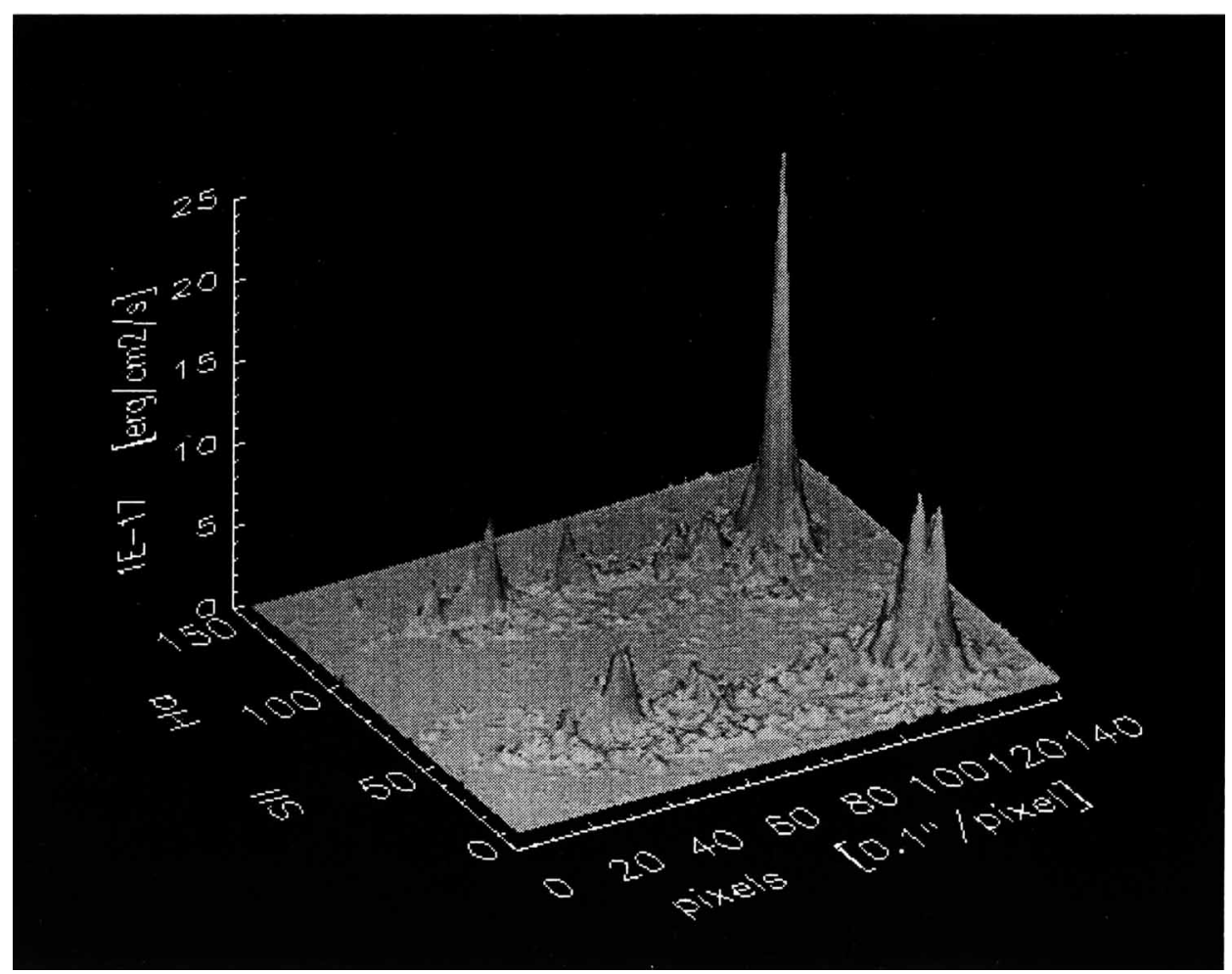

Figure 3. Intensity along the jet in $\mathrm{H} \alpha$ and $[\mathrm{SII}]$ as $3 \mathrm{D}$ plots. Images have been smoothed to 0.1 arcsec resolution, and have been rotated to match the orientation in Figure 1.

the ground based picture, in that it has a much more irregular appearence with a small bright core instead of the smooth elliptic, 'bow-shock' like shape visible from the ground. In Figure 3 we display the $\mathrm{H} \alpha$ and the [SII] data as 3-D plots. These images have been smoothed to a resolution of 0.1 arcsec. On this image, as well as in the raw data one can clearly see two jets. One brighter towards the north, and one fainter towards the south. It is also obvious that feature $\mathrm{D}$ is much brighter in $\mathrm{H} \alpha$ than in [SII]. There is also more structure in the latter. To display this we show contour plots of the line emission images in Figure 4, expanded so that only the 2 arcsec by 2 arcsec surrounding knot $\mathrm{D}$ are shown. The inherent resolution in these plots is $6.5 \mathrm{AU}$.

We have measured the cross section of the jet along its extent and we find that there is no apparent expansion of the visible material. The FWHM is found to be almost constant at $0.42 \pm 0.13$ arcsec from where the signal/noise becomes high enough to get a reasonable measurement, until it 'disappears' into knot D. This corresponds to a 'jet-diameter' of 

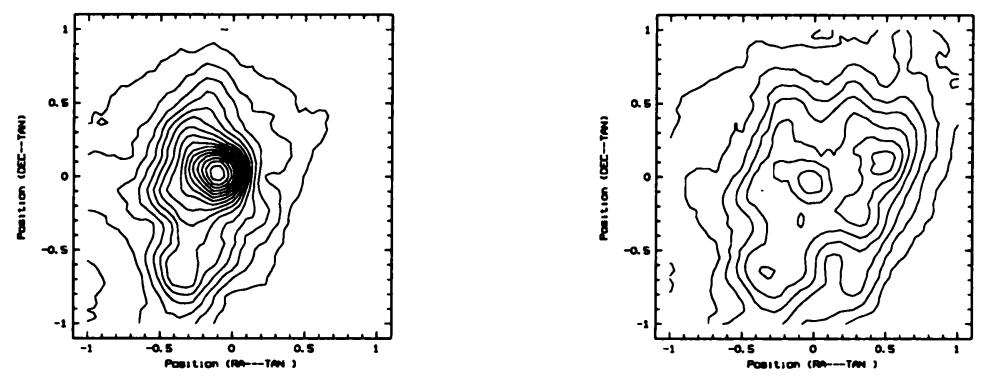

Figure 4. $\mathrm{H} \alpha$ and [SII] contour representations of knot D. Offsets are in arcseconds, and the direction towards IRS5 is in the upper left.

$60 \mathrm{AU} \pm 20 \mathrm{AU}$. As mentioned above, and as can be seen in Figure 2, the brighter portion of the jet 'wiggles' as it progresses outwards. If one plots the peak brightness of the jet, one can see that the area on the sky onto which this 'wiggling' takes place, is cone-shaped with the apex just where the jet first emerges (with a FWHM of 0.4 arcsec). The cone defined by the 'jet-wiggling' expands to $\approx 2$ arcsec just as the jet reaches knot $D$. This could conceivably be representative of the projected shape of a cavity within which the jet moves. The nodes defined by the undulation of the jets are separated by $\approx 500 \mathrm{AU}$ or $7.5 \times 10^{15} \mathrm{~cm}$. The opening angle defined by the expanding envelope is $15^{\circ}$. Comparing the $\mathrm{H} \alpha$ data with the I-band image in Figure 2 we see that the well collimated jet emerges out of a cone shaped reflection nebula which possesses an opening angle of $70^{\circ}$. This cone immediately starts to bend 'inwards' towards the jet axis on both edges, and after only about 1.1 arcsec it appears to have become 'collimated', and there are two bright, more or less parallell streams with a separation of 1.3 arcsec. These features are all seen superposed on top of a fainter nebulosity with a larger opening angle $\left(\approx 150^{\circ}\right)$, which is identical to the cone shaped nebula seen in ground based data (e.g. Fridlund \& Liseau, 1994, Figures 1 c \& 1d), and which is assumed to trace the molecular outflow cavity. The emission from the cone shaped nebula is almost certainly pure reflection, since no trace of it is visible on either the $\mathrm{H} \alpha$ or the [SII] images - each of which traces high excitation (high velocity shocks) and low excitation (low velocity shocks) respectively. The same is true for a bright spot (diameter $\approx 0.3 \mathrm{arcsec}$ ) which is visible in the I-band image (and very faintly also in the R-band data) very near the apex of the cone shaped nebula. Although very tentative, a possible interpretation of this signature is that it is due to light reflected from a rift in the extinction towards IRS5.

No crossing shocks are immediately evident from the $\mathrm{H} \alpha$ and the [SII] 


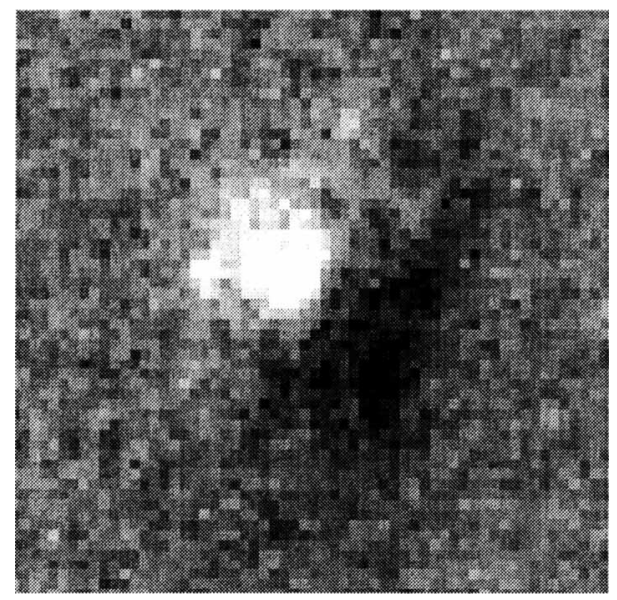

Figure 5. Difference between $\mathrm{H} \alpha$ (white) and [SII] (black) images for the bright feature in knot D. The area displayed is $1^{\prime \prime} \times 1^{\prime \prime}$. The direction towards IRS5 is in the upper left

images. A careful examination of the emission maxima show that the ionic species are slightly displaced from each other, and positioned close to the 'nodes' mentioned above. This suggest an interpretation where they actually represent shocks at the edge of the cavity walls, which arise due to interaction with the undulating jet.

The feature called knot $\mathrm{D}$ is resolved into one very bright $\mathrm{H} \alpha$ peak and several fainter [SII] intensity maxima, each of which have a FWHM of 0.3 arcsec (see Figure 4). It is seen that what was interpreted as a bow shock in the ground based data now is only the fainter envelope surrounding these peaks. The single bright core seen in the $\mathrm{H} \alpha$ image, clearly has the shock on the side away from IRS5, as defined by the steepness of the intensity gradient. Taking the difference between the $\mathrm{H} \alpha$ and [SII] we can clearly see (Figure 5) that they are well separated with [SII] on the outside (relative to IRS5). This would indicate that the Mach disk is the shock with the higher excitation, and thus that the jet is less dense than the ambient material that it encounters. On the other hand, the steep gradient on the side away from IRS5 is indicative of a jet denser than the ambient medium. The elongation of the bright $\mathrm{H} \alpha$ feature has its long axis well pointed towards IRS5.

\section{Conclusions}

We have observed the jet emanating from L1551 IRS5 with the Hubble Space Telescope. We find that the brightest knot has a pronounced small scale structure, and that those small features $(\leq 40 \mathrm{AU})$ have a high excitation character. The shock is located on the side furthest away from the 
originating source on the Mach disk, but the highest excitation is to be found on the side of the Mach disk towards IRS5.

At the origin of the visible jet, it emerges already well collimated to a diameter of $\approx 40 \mathrm{AU}$. It appears to 'wiggle' within a cavity of diameter $\approx 100 \mathrm{AU}$, until it reaches the assumed 'working surface', i.e. knot D. The nodes of the undulation of the jet are separated along the jet axis by $\approx 500 \mathrm{AU}$.

Acknowledgements: The authors gratefully acknowledge the support of STSCI staff. We are pleased to acknowledge the excellent arrangements for the symposium in Chamonix, and finally MF thanks the organisers for the opportunity to present these results.

\section{References}

Beichman, C., Harris, S., 1981 ApJ 245, 589

Bieging, J.H., Cohen, M., Schwartz, R.D., 1984, ApJ 282, 699

Bieging, J.H., Cohen, M., 1985 ApJ 289, L5

Carr, J.S., Harvey, P.M., Lester, D.F. 1987 ApJ 321, L71

Cohen, M., Bieging, J.H., Schwartz, R.D., 1982, ApJ 253, 707

Cudworth, K.M., Herbig, G., 1979 AJ 84, 548

Curiel, S., 1995 RevMexAASC Series de conferencias, volumen 1 abril 1995, p59

Elias, J., 1978 ApJ 224, 857

Felli, M., Perinotto, M., 1974 Ap\&SS 26, 11

Fridlund, C.V.M., Nordh, H.L., van Duinen, R.J., Aalders, J.W.G., Sargent, A.I., 1980 A\&A 91, L1

Fridlund, C.V.M., Sandqvist, Aa., Nordh, H.L., Olofsson, G., 1989 A\&A 213, 310

Fridlund, C.V.M., Knee, L.B.G., 1993 A\&A 268, 245

Fridlund, C.V.M., Liseau, R., 1994 A\&A 292, 631

Heathcote, S., et al., 1996, AJ 112, 1141

Herbig, G.H., 1974 Lick Obs. Bull., 658

Holtzmann, J.A., et al., 1995, PASP, 107, 1065

Kenyon, S.J., Gomez, M., Marzke, R.O., Hartmann, L., 1994 AJ 108, 251

Knapp, G.R., Kuiper, T.B.H., Knapp, S.L., Brown, R.L., 1976 ApJ 206, 713

Luyten, W.J., 1963 Harvard Annu. Card No. 1589

Luyten, W.J., 1971 The Hyades, Univ. Minnesota P., Minneapolis

Lynds, B.T., 1962 ApJS 7, 1

Mundt, R., Fried, J.W., 1983, ApJ, 274, L83

Mundt, R., Stocke, J., Strom, S.E., Strom, K.M., Anderson, E.R., 1985 ApJ 297, L41

Neckel, Th., Staude, H.J., 1987 ApJ 322, L27

Reipurth, B., 1994, A general catalogue of Herbig-Haro objects, electronically published via anon. ftp to ftp.hq.eso.org, directory /pub/Catalogs/Herbig-Haro.

Sandqvist, Aa., Bernes, C., 1980, A\&A 89, 187

Sharpless, S., 1959 ApJS 4, 257

Snell, R.L., Loren, R.B., Plambeck, R.L., 1980 ApJ 239, L17

Staude, H.J., Elsässer, H., 1993 A\&A Review. 5, 165

Strom, S.E., Grasdalen, G.L., Strom, K.M., 1974, ApJ, 191, 111

Strom, S.E., Strom, K.M., 1974, Vrba, F.J., 1976, AJ, 81, 320

Trauger, J.T., et al., 1994, ApJ 435, L3 